\title{
Efficacy and Safety of the use of Pertuzumab in Metastatic Breast Cancer Patients in a Real-World Setting: Results from the SUPER-GONO (Gruppo Oncologico del Nord Ovest) Study
}

Ornella Garrone ( $\nabla$ ornella.garrone@gmail.com )

Azienda Ospedaliera S Croce e Carle, Ospedale di Insegnamento Cuneo https://orcid.org/0000-00017359-5410

\section{Tommaso Giarratano}

Istituto Oncologico Veneto Istituto di Ricovero e Cura a Carattere Scientifico

\section{Eva Blondeaux}

Ospedale Policlinico San Martino Istituto di Ricovero e Cura a Carattere Scientifico per I'Oncologia:

Ospedale Policlinico San Martino

\section{Loretta D'Onofrio}

Policlinico Universitario Campus Bio-Medico

\section{Andrea Michelotti}

AOU Pisana: Azienda Ospedaliero Universitaria Pisana

\section{Alessandra Beano}

Azienda Ospedaliero Universitaria Citta della Salute e della Scienza di Torino

\section{Giuseppe Aprile}

Ospedale San Bortolo di Vicenza

\section{Valentina Guarneri}

University of Padova: Universita degli Studi di Padova

\section{Matteo Paccagnella}

Azienda Ospedaliera S. Croce e Carle Ospedale di Insegnamento Cuneo

\section{Paola Vanella}

Azienda Ospedaliera S. Croce e Carle Ospedale di Insegnamento Cuneo

Chiara Saggia

Azienda Ospedaliero Universitaria Maggiore della Carita

\section{Luigi Coltelli}

Azienda USL Toscana nord ovest Sede di Livorno

\section{Marina E Cazzaniga}

Ospedale San Gerardo

\section{Nicla La Verde}

Ospedale Luigi Sacco: Ospedale Luigi Sacco-Polo Universitario 


\section{Fiorella Ruatta}

Azienda Ospedaliera S Croce e Carle Ospedale di Insegnamento Cuneo

\section{Elena Collovà}

ASST Ovest Milanese: Aziende Socio Sanitarie Territoriale Ovest Milanese

\section{Filippo Montemurro}

Candiolo Cancer Institute, FPO-IRCCS

\section{Lucia Del Mastro}

Ospedale Policlinico San Martino Istituto di Ricovero e Cura a Carattere Scientifico per I'Oncologia:

Ospedale Policlinico San Martino

\section{Elisabetta Landucci}

AOU Pisana: Azienda Ospedaliero Universitaria Pisana

\section{Giacomo Allegrini}

Azienda USL 6: Azienda USL Toscana nord ovest Sede di Livorno

\section{Daniele Santini}

Policlinico Universitario Campus Bio-Medico

\section{Livio Blasi}

Ospedale Civico Benfratelli: Ospedale Civico Palermo

\section{Raffaele Ardito}

IRCCS CROB: Istituto di Ricovero e Cura a Carattere Scientifico Centro di Riferimento Oncologico della Basilicata

\section{Nerina Denaro}

Azienda Ospedaliera S Croce e Carle Ospedale di InsegnamentoCuneo

\section{Enrico DeConciliis}

Ospedale Cardinal Massaia di Asti

\section{Mario Airoldi}

Azienda Ospedaliero Universitaria Citta della Salute e della Scienza di Torino

\section{Marco C Merlano}

Candiolo Cancer Institute, FPO-IRCCS

\section{Research Article}

Keywords: Pertuzumab and Trastuzumab, Taxanes, HER2, Advanced breast cancer, Real-world

Posted Date: April 14th, 2021

DOI: https://doi.org/10.21203/rs.3.rs-409228/v1

License: (a) (1) This work is licensed under a Creative Commons Attribution 4.0 International License. Read Full License 


\section{Abstract}

Background: Real world data have the potential to demonstrate the applicability of the results of randomized studies in the general population. SUPER trial was conducted in order to assess the activity, the efficacy and the safety of the combination of pertuzumab, trastuzumab and chemotherapy in clinical practice.

Material and methods: Patients diagnosed with HER2 positive metastatic breast cancer (mBC) and treated with pertuzumab, trastuzumab and chemotherapy were accrued at 18 italian hospitals. Data were retrospectively collected in the time frame between pertuzumab availability in clinical practice and study approval in 2016, and prospectively collected thereafter.

Results: Overall 342 HER2 positive $\mathrm{mBC}$ were accrued. 172 patients had relapsed disease and $56.4 \%$ of them received neo/adjuvant trastuzumab. 205 patients received docetaxel. Objective response rate was 76.3\% (95\%Cl: 71.4-80.7). Median progression free survival (PFS) and overall survival (OS) were 24.3 months (95\% Cl: $20.0-28.9)$ and 70.2 months (95\% Cl: 61.4-79.0) respectively. Triple positive patients treated with endocrine therapy in addition to pertuzumab and trastuzumab maintenance had a significant longer PFS and OS than patients who did not. mPFS was 31.2 months and 13 months respectively $(\mathrm{HR}=0.47 ; 95 \% \mathrm{Cl}: 0.33-0.66 ; p<0.001)$ and mOS was 72.3 months and 56.8 months respectively $(\mathrm{HR}=0.58 ; 95 \% \mathrm{Cl}: 0.36-0.92 ; \mathrm{p}=0.02)$. Pretreatment with trastuzumab did not hamper the outcome. In addition, maintaining the dual blockade inhibition at disease progression with the same CT partner or alternative endocrine agent leading to further benefit.

Conclusions: SUPER suggests that results of first-line treatment with pertuzumab, trastuzumab and chemotherapy in unselected patients are consistent with findings from CLEOPATRA trial.

Moreover, as expected from real world evidence, new insights have emerged.

\section{Introduction}

HER2 positive tumors represent about $15 \%$ of all breast cancer diagnoses and are characterized by higher aggressiveness and worse prognosis compared to other subtypes [1,2]. The introduction of trastuzumab has significantly improved the outcome of HER2 positive breast cancer patients both in the adjuvant and advanced setting [3-8]. Pertuzumab, a humanized monoclonal antibody that binds to the extracellular domain II of HER2, further improved the outcome of metastatic breast cancer patients (mBC). In the CLEOPATRA randomized phase III study trial patients previously untreated for advanced disease were randomly assigned to receive the combination of docetaxel, trastuzumab and pertuzumab or docetaxel trastuzumab and placebo. The dual HER2 blockade plus CT significantly increased both progression free survival (PFS) and overall survival (OS) [9-11]. Pertuzumab and trastuzumab combination was also evaluated in a small phase II trial in which docetaxel was substituted with paclitaxel and in the larger PERUSE trial, in which anti HER2 therapy was associated with paclitaxel, docetaxel or nab-paclitaxel [1214]. The addition of pertuzumab to trastuzumab and paclitaxel showed remarkable outcome results with 
reduced toxicity in comparison to docetaxel. However, in the CLEOPATRA trial, a minority of patients had received adjuvant trastuzumab, which is now the standard treatment for the vast majority of HER2 + early breast cancer.

SUPER is a retrospective and prospective observational study collecting data of HER2 positive metastatic breast cancer patients treated with chemotherapy, trastuzumab and pertuzumab in a real-world setting, aimed at evaluating whether the CLEOPATRA results are reproducible outside the context of a clinical trial.

\section{Methods}

The SUPER trial is an observational, retrospective and prospective multicenter study aimed at evaluating the efficacy and safety of the association of CT plus trastuzumab and pertuzumab in a real-world setting, carried out at 18 Italian hospitals.

\section{Aims}

Study Design

The primary aim was the evaluation of the efficacy of pertuzumab based treatment, progression free survival (PFS) was the primary study endpoint. Secondary aims included: 1) the activity - namely the objective response rate (ORR), the disease control rate (DCR), and the clinical benefit rate (CBR); 2 ) the outcome in pre-defined subsets of patients according to prior adjuvant trastuzumab and endocrine therapy maintenance during dual blockade maintenance; 3 ) the safety of the combination; and 4) the overall survival (OS).

\section{Patients}

All patients treated with a combination of CT, trastuzumab and pertuzumab since the availability of pertuzumab in Italy (2012) were enrolled. Data were retrospectively collected in the time frame between pertuzumab availability and study approval in 2016, and prospectively collected thereafter. Docetaxel and paclitaxel were administered at 75 or $100 \mathrm{mg} / \mathrm{m} 2$ intravenously every 3 weeks, and $80 \mathrm{mg} / \mathrm{m} 2$ intravenously weekly, respectively. Notably, 3 administration of weekly paclitaxel were considered as 1 cycle of chemotherapy. Pertuzumab and trastuzumab were administered intravenously every 3 weeks at their approved doses (pertuzumab $840 \mathrm{mg}$ loading dose at the first cycle, $420 \mathrm{mg}$ at following cycles, trastuzumab $8 \mathrm{mg} / \mathrm{kg}$ loading dose at the first cycle and $6 \mathrm{mg} / \mathrm{kg}$ at subsequent cycles). Treatment was continued until disease progression, unacceptable toxicity or patient refusal. Chemotherapy dose reductions or cycles delays were reported. Tumor response was assessed using the Response Evaluation Criteria in Solid Tumors (RECIST) criteria version 1.1. [15]. every 12 weeks according to rules of the Agenzia Italiana del Farmaco (AIFA) registry. Toxicity was evaluated according to National Cancer Institute Common Terminology Criteria for Adverse Events (NCl-CTCAE, version 4). All patients underwent 
echocardiogram for left ventricular ejection fraction (LVEF) evaluation at baseline and every 3 months as per indication. The study was approved by local Ethical Committee of participating Institutions. All patients signed an informed consent.

\section{Statistical analyses}

The primary endpoint was the evaluation of the PFS defined as the time elapsed between the first cycle of therapy and disease progression or death from any cause, whichever occurred first, or at the date of the last follow-up for censored patients. Secondary endpoints included OS, defined as the time elapsed between the first cycle of therapy and death from any cause, or the date of the last follow-up for censored patients. ORR was defined as the amount of complete response (CR) and partial response (PR). DCR was defined as the sum of CR, PR and disease stabilization (SD). CBR was defined as the occurrence of CR, PR or long-lasting ( $\geq 24$ weeks) disease stabilization. The PFS and OS were estimated based on the Kaplan-Meier method [16]. The Log-rank test [17] was used to compare PFS differences in prespecified subsets of patients. A p value lower or equal than 0.05 was considered as significant in all statistical analyses.

\section{Results}

From March 2016 to February 2019, 347 HER2 positive metastatic breast cancer patients treated with the combination of CT, pertuzumab and trastuzumab were accrued. Five patients were excluded from the evaluation due to incomplete data. Therefore, the final analyses were conducted on 342 patients, 236 patients $(69 \%)$ in the retrospective cohort and 106 patients (31\%) in the prospective cohort. The main patients' characteristics are summarized in Table 1. 
Table 1

Patients' characteristics

\begin{tabular}{|c|c|}
\hline & $\begin{array}{l}\text { Patients N. } 342 \\
\text { (\%) }\end{array}$ \\
\hline \multicolumn{2}{|l|}{ Age- $y r$} \\
\hline Median (range) & $55(19-80)$ \\
\hline \multicolumn{2}{|l|}{ Disease status at study entry } \\
\hline Relapsed disease & $172(50.3)$ \\
\hline De novo disease & $170(49.7)$ \\
\hline \multicolumn{2}{|l|}{ ECOG performance status } \\
\hline 0 & $269(78.7)$ \\
\hline 1 & $54(15.8)$ \\
\hline 2 & $19(5.5)$ \\
\hline Median (range) & $0(0-2)$ \\
\hline HER2 status assessed by IHC or FISH & $342(100)$ \\
\hline $\begin{array}{l}\text { Hormone Receptor status at study } \\
\text { entry } \\
\text { Positive } \\
\text { Negative }\end{array}$ & $\begin{array}{l}235(68.7) \\
107(31.3)\end{array}$ \\
\hline $\begin{array}{l}\text { Hormone receptor status at } \\
\text { diagnosis* } \\
\text { Positive } \\
\text { Negative } \\
\text { Unknown }\end{array}$ & $\begin{array}{l}130(75.6) \\
40(23.2) \\
2(1.2)\end{array}$ \\
\hline \multicolumn{2}{|l|}{ Neo/Adiuvant therapy* } \\
\hline CT alone & $50(29.1)$ \\
\hline $\mathrm{CT}+$ trastuzumab & $97(56.4)$ \\
\hline No & 25 (14.5) \\
\hline
\end{tabular}

*Data on 172 patients; ** On $129 \mathrm{HR}$ + patients at diagnosis CNS: Central Nervous System 


\begin{tabular}{|c|c|}
\hline & $\begin{array}{l}\text { Patients N. } 342 \\
(\%)\end{array}$ \\
\hline \multicolumn{2}{|c|}{ Adjuvant endocrine therapy ** } \\
\hline Yes & $120(93.0)$ \\
\hline No & $9(7.0)$ \\
\hline \multicolumn{2}{|c|}{ Metastatic disease } \\
\hline Visceral & $219(64.0)$ \\
\hline Non visceral & $97(28.4)$ \\
\hline Both & $150(43.9)$ \\
\hline \multicolumn{2}{|l|}{ Metastatic sites } \\
\hline 1 & $85(24.8)$ \\
\hline 2 & $83(24.3)$ \\
\hline 3 & $81(23.7)$ \\
\hline$\geqslant 4$ & $93(27.2)$ \\
\hline Median (range) & $3(1-8)$ \\
\hline \multicolumn{2}{|c|}{ Most common metastatic sites } \\
\hline Bone & $180(52.6)$ \\
\hline Liver & $140(41.0)$ \\
\hline Lung & $108(31,6)$ \\
\hline Soft Tissue & $247(72.2)$ \\
\hline Pleura & $27(7.9)$ \\
\hline CNS & $14(4.1)$ \\
\hline \multicolumn{2}{|c|}{${ }^{*}$ Data on 172 patients; $* \star$ On $129 \mathrm{HR}+$ patients at diagnosi } \\
\hline CNS: Central NeI & \\
\hline
\end{tabular}

Briefly, the median age was 55 years (range 19-80), the majority of patients had good performance status and about two third of them had hormone receptor positive disease. Visceral disease was present in 219 patients (64\%) and the median number of metastatic sites was 3 (range: 1-8). Fourteen patients (4.1\%) presented central nervous system (CNS) involvement. 170 patients $(49.7 \%)$ had de novo metastatic disease and 97 of 172 patients (56.4\%) with relapsed disease had received prior neo/adjuvant trastuzumab. 58 patients (17\%) were 70 years or older: among them, visceral disease was present in 32 patients (55.2\%), while 20 patients (34.5\%) had both visceral and non-visceral disease and $25(43.1 \%)$ had non visceral involvement only. 
The median disease-free interval (DFI) was 56.9 months (range, 3.6-286.8 months). Regarding the type of CT, 205 patients (59.9\%) were treated with docetaxel, 136 (39.8\%) received weekly paclitaxel and 1 patient received vinorelbine. In particular, weekly paclitaxel was the chemotherapy backbone in the majority $(72.4 \%)$ of patients aged $>70$ years.

Overall, 2,360 cycles of chemotherapy were administered, and the patients were exposed to a median of 6 cycles (range: $1-24)$. The CT dose was reduced in 177 cycles (7.5\%) and 48 patients (14\%) needed a dose reduction mainly due to toxicity. The treatment was delayed in 49 cycles $(2.1 \%)$ and in 34 patients $(9.9 \%)$ mainly due to: (1) toxicity (24 patients, $7 \%$, including 3 pts with asymptomatic decline of left ventricular ejection fraction); (2) patient request (6 patients, 1.7\%); (3) bone radiotherapy (2 patients, $0.6 \%)$; (4) flu-like syndrome (2 patients, $0.6 \%$ ). At the end of the planned CT, 320 patients $(93.5 \%)$ underwent maintenance with pertuzumab and trastuzumab. The median duration of the maintenance therapy was 24 months (range: 1.4-78.1). Overall, 235 patients (68.7\%) had triple positive disease, 177 (75.3\%) received maintenance hormonal therapy along with pertuzumab and trastuzumab at the time of chemotherapy discontinuation.

\section{Activity}

Overall, the ORR and CBR in the whole population were $76.3 \%(95 \% \mathrm{Cl}: 71.4-80.7)$ and $90.9 \%(95 \% \mathrm{Cl}$ : 87.4-93.8), respectively (Table 2). The ORR related to the different companion drug was $78.5 \%(95 \% \mathrm{Cl}$ $72.3-84.0)$ and $72.8 \%(95 \% \mathrm{Cl}: 64.5-80.1)$ with docetaxel and weekly paclitaxel, respectively $(p=0.22)$. However, in the 172 relapsed patients the ORR was $69.8 \%$ (95\% Cl: $63.3-76.5)$, and was $82.9 \%(95 \% \mathrm{Cl}$ : $76.4-88.3)$ in patients with de novo metastatic disease $(p=0.006)$. In the elderly cohort, the ORR was $67.2 \%$ (95\%Cl: 53.7-79.0).

Table 2

Activity

\begin{tabular}{|ll|}
\hline Response & Patients N. 342 (\%) \\
\hline Objective response & $261(76.3)$ \\
\hline Complete response & $79(23.1)$ \\
\hline Partial response & $182(76.3)$ \\
\hline Stable disease & $52(15.2)$ \\
\hline Progressive disease & $24(7.0)$ \\
\hline Not evaluable & $5(1.5)$ \\
\hline Clinical benefit rate & $309(90.3)$ \\
\hline Disease control rate & $313(91.5)$ \\
\hline
\end{tabular}

\section{Efficacy}


At a median follow-up of 40.8 months (1.1-94.7 + months), 234 PFS events and 124 deaths have been recorded. The median PFS in the whole population was 24.3 months ( $95 \% \mathrm{Cl}$ : 20.0-28.9). The median OS in the entire cohort was 70.2 months (95\% Cl: 61.4-79.0) (Fig. 1). In patients who received prior neo/adjuvant trastuzumab, the median PFS and OS were 19.0 months (95\% Cl: 13.7-24.3) and 59.7 months (95\% Cl: $39.8-79.6)$, respectively, demonstrating the efficacy of the treatment also in this pretreated population. However, a significant difference in the median PFS and OS was observed among patients with prior exposure to trastuzumab compared to trastuzumab naïve patients, being 28.7 months (95\% Cl: $21.7-38.7 ; \mathrm{HR}=0.64 ; 95 \% \mathrm{Cl}: 0.49-0.85 ; \mathrm{p}=0.002)$ and not reached, respectively, in the latter group (HR $=0.69 ; 95 \% \mathrm{Cl}: 0.48-0.98 ; p=0.04)$ (Fig. 2). Furthermore, we detected significantly longer PFS and OS in patients with triple positive disease exposed to the combination of endocrine therapy, pertuzumab and trastuzumab as maintenance therapy compared to patients who did not receive hormone therapy. The median PFS was 31.2 months (95\% Cl: $24.9-37.5)$ in the cohort of patients treated with endocrine therapy in addition to dual blockade maintenance compared to 13.0 months $(95 \% \mathrm{Cl}$ : $10.1-15.9)$ in those patients who received only pertuzumab and trastuzumab $(\mathrm{HR}=0.47 ; 95 \% \mathrm{Cl}$ : $0.33-$ $0.66 ; \mathrm{p}<0.001)$. The Median OS was 72.3 months $(95 \% \mathrm{Cl}$ not reached) and 56.8 months $(95 \% \mathrm{Cl}$ not reached) in the two groups, respectively $(\mathrm{HR}=0.58 ; 95 \% \mathrm{Cl}: 0.36-0.92 ; \mathrm{p}=0.02)$.

No significant difference was observed between paclitaxel and docetaxel in terms of PFS and OS.

\section{Safety}

Adverse events are reported in Table 3. No new safety concerns about the association of CT plus pertuzumab and trastuzumab emerged from our cohort of real-life patients. The most frequent adverse events were asthenia, diarrhea and mucositis, mostly grade 1 or 2 . Thirty-two patients $(9.3 \%)$ experienced cardiac toxicity, which led to discontinuation of anti HER2 therapy in 17 of them (4.9\%). Twenty-three patients $(6.7 \%)$ had LVEF decline and 14 of them (4.1\%) were symptomatic. 
Table 3

Adverse Events

\begin{tabular}{|c|c|c|c|}
\hline \multirow[t]{2}{*}{ Advers Events } & All grades & Grade 3 & Grade 4 \\
\hline & No. (\%) & No. (\%) & No. (\%) \\
\hline Leucopenia & $84(24.6)$ & $15(4.4)$ & $3(0.9)$ \\
\hline Neutropenia & $90(26.6)$ & $8(2.3)$ & $18(5.3)$ \\
\hline Febrile Neutropenia & $11(3.2)$ & $11(3.2)$ & - \\
\hline Anemia & $106(31)$ & $3(0.9)$ & - \\
\hline Diarrhea & $162(47.4)$ & $11(3.2)$ & $1(0.3)$ \\
\hline Asthenia & $212(62)$ & $6(1.7)$ & - \\
\hline Mucosal inflammation & $110(32.2)$ & $2(0.6)$ & - \\
\hline Nausea & 39 (11.4) & $4(1.2)$ & - \\
\hline Peripheral neuropathy & $135(39.5)$ & $5(1.5)$ & - \\
\hline Arthro-myalgia & $26(7.6)$ & - & - \\
\hline Rash & $21(6.1)$ & - & - \\
\hline H-F Syndrome & $50(14.6)$ & $4(1.2)$ & - \\
\hline Alopecia & $172(50.3)$ & - & - \\
\hline Dyspnea & $20(5.8)$ & $5(1.5)$ & - \\
\hline ALT increase & $43(12.6)$ & - & $1(0.3)$ \\
\hline AST increase & $46(13.5)$ & $2(0.6)$ & - \\
\hline Nail disorders & $33(9.6)$ & $2(0.6)$ & - \\
\hline Pruritus & $13(3.8)$ & - & - \\
\hline Cardiac toxicity & $31(9.1)$ & $16(4.7)$ & - \\
\hline Left ventricular ejection fraction drop & $23(6.7)$ & $11(3.2)$ & - \\
\hline
\end{tabular}

\section{Long term outcomes}

At the time of the present analysis (October 2020), 89 patients (26\%) were still on pertuzumab and trastuzumab maintenance. Fifty-five of them (16\%) were receiving endocrine therapy together with monoclonal antibodies, and 24 patients (7\%) discontinued pertuzumab during maintenance due to toxicity. Overall, HER2 double blockade maintenance was discontinued in 253 patients (74\%). 231 patients (67.5\%) experienced disease progression, 21 (6.1\%) discontinued dual blockade maintenance and 1 patient was receiving maintenance with trastuzumab alone. These patients were actually free from 
progression. Apart from progression, the reasons for discontinuation of the maintenance at any time included persistent and symptomatic drop of LVEF (12 patients, 3.5\%), toxicity (13 patients, 3.8\%), medical decision (9 patients, $2.6 \%$ ), refusal or patient decision (7 patients, $2 \%)$, stroke (2 patients, $0.6 \%$ ), deterioration of PS (2 patients, $0.6 \%$ ), diagnosis of acute lymphatic leukemia ( 1 patient, $0.3 \%$ ) and death (1 patient, $0.3 \%$ ). Of these latter group of patients 25 patients went on disease progression. At disease progression all but 18 patients $(7.8 \%)$ were given second line therapy. 145 patients $(62.8 \%)$ received TDM1, 22 (9.5\%) the association of lapatinib and capecitabine, 16 (6.9\%) trastuzumab plus chemotherapy or endocrine therapy, and $17(7.3 \%)$ were treated with CT or were enrolled in clinical trials. 6 patients (2.6\%) who experienced CNS progression as unique site of disease were treated with local therapy and went on maintenance with pertuzumab and trastuzumab. Interestingly, 7 patients (3\%) who had developed metastases outside the CNS underwent rechallenge with docetaxel plus monoclonal antibodies or crossing to another endocrine therapy plus HER2 dual blockade. All of them benefited again from the therapy.

\section{Discussion}

Our retrospective and prospective analysis performed on patients from a real-world setting confirms the findings of the CLEOPATRA trial in terms of ORR, PFS and OS, with no new safety concerns. Most importantly, additional information not clearly reported in the pivotal study emerges from our analysis. First, in our series, triple positive patients received endocrine therapy in association with pertuzumab and trastuzumab maintenance, which was not allowed in the CLEOPATRA trial. This combination led to better efficacy results in patients who underwent both endocrine and anti HER2 maintenance therapy in comparison to endocrine responsive patients treated with anti HER 2 therapy alone, as demonstrated by a significantly longer median PFS in the former group (31.2 vs. 13.0 months, respectively, $p<0.001)$. This advantage resulted in longer OS (72.4 months $v s .65 .8$ months, respectively, $\mathrm{p}=0.01)$. Given the crosstalk between HER2 and ER, HER2 suppression might strengthen and restore the ER pathway [18]. Therefore, the co-administration of endocrine therapy with HER2 blockade contributes to a more profound inhibition leading to better outcome [18-20]. Other authors have reported similar findings in clinical practice [21,22], underlining the importance of the inhibition of the ER pathway in HR-positive HER2 positive $\mathrm{mBC}$. This issue is also supported by the 5th ESO-ESMO international consensus guideline for advanced breast cancer ABC-5 [23].

Second, in our cohort, 172 patients had relapsed disease and 97 of them (56.4\% of the relapsed population and $28.4 \%$ of the entire group) received trastuzumab as part of their adjuvant treatment. Despite prior exposure to trastuzumab seemed not to affect subsequent therapy with pertuzumab and trastuzumab, the median PFS and OS in trastuzumab naïve patients were significantly longer than in patients exposed to adjuvant trastuzumab. In CLEOPATRA, only 47 patients $(11.7 \%)$ treated with dual blockade received trastuzumab as neo/adjuvant treatment [9]. No negative findings were described in the trial, however, the small number of patients exposed to adjuvant trastuzumab limited the applicability of the results to the general patient population that is usually exposed to therapy with trastuzumab in the early setting. Gamucci et al. [22] reported a non-significant difference in the median PFS between patients 
naïve and exposed to prior trastuzumab (23 and 17 months, respectively, $p=0.10$ ), however a significant longer OS was observed in the trastuzumab naïve group $(p=0.02)$. Another report did not describe a negative impact of neo/adjuvant trastuzumab on the efficacy of pertuzumab. However, no difference in the outcome between the two groups was observed ${ }^{21}$.

Third, as reported by others $[12,14,21,22,24,25]$, the CT backbone has not a significant influence on the outcome. In the advanced setting, clinicians should choose the less toxic agent in order to preserve patients' quality of life. To this aim, paclitaxel may be preferable, as demonstrated by the PERUSE trial ${ }^{14}$. Moreover, the duration of CT does not hamper the outcome [26].

An intriguing issue coming out from our analysis is the rechallenge with the same CT partner or with alternative endocrine agent at disease progression. This strategy allows to prolong the inhibition with dual blockade while maintaining activity, leading to a delay in the use of subsequent therapy exploiting completely the performance of the drug. This aspect deserves further research.

Finally, there is uncertainty about the direct transferability of clinical trial results in a real-world setting, due to the strict patient selection criteria and the difficulty to apply them to the general patient population. In contrast, real-world studies include a wide proportion of patients who are excluded from registration studies, such as patients with brain metastases. Therefore, real-world studies might mimic phase IV trials. In the past, many real-word studies have been conducted to confirm data of pivotal phase III studies [2729].

One important limitation of our study is its observational nature, albeit one third of our population was treated prospectively. Response evaluation, in clinical practice, could not be properly evaluated. Tumor assessment was evaluated every 3 months accordingly to AIFA (Agenzia Italiana Farmaco) registry rules. On the other hand, our study also has some clear strengths, as it supports the use of endocrine therapy in addition to anti HER2 maintenance in hormone receptor positive patients and reinforces the available data regarding activity and efficacy in patients previously exposed to adjuvant trastuzumab. Moreover, it suggests the possibility to maintain dual blockade inhibition at disease progression with the same CT partner or changing endocrine agent.

\section{Conclusions}

In conclusion, the SUPER study exemplifies the utility of real-life studies and, to the best of our knowledge, it is the largest real-life study in HER2 positive metastatic breast cancer to provide evidence that the treatment with taxane plus pertuzumab and trastuzumab is feasible, active and effective, without any additional toxicity in unselected patients.

\section{Declarations}

\section{Funding: NA}




\section{Conflict of interest}

O.G. reports acting as consultant for Novartis, Eisai, MSD, Eli Lilly and receiving honoraria from Pfizer, Novartis. A.M. reports acting as consultant for Novartis, Gentili, Pfizer, Eli Lilly, Novartis. G.A. reports honoraria from Roche, Baxter, Servier, Amgen, Eli Lilly, Merck Serono. V.G. reports acting as consultant for Eli Lilly, Novartis, Roche, MSD and receiving honoraria from Eli Lilly, Novartis. N.LV. reports research funding from Eisai, acting as consultant for MSD, Roche, Novartis and receiving honoraria from GSK, reports receiving support for congress participation from Pfizer, Gentili, Celgene. F.M. reports acting as consultant for Roche, SeaGen and receiving honoraria from Roche, Novartis, Pfizer, Eli Lilly, Pierre Fabre, reports receiving support for congress participation from Roche. L.D.M. reports acting as consultant for Roche, Eli Lilly, Novartis, MSD, Genomic Health, Pierre Fabre, Daiichi Sankyo, Seagen, AstraZeneca, Eisai and receiving honoraria from Roche, Novartis, Eli Lilly, MSD, Pfizer, Ipsen, reports receiving support for congress participation from Roche, Pfizer, Celgene. E.L. reports acting as consultant for Novartis, Gentili, Pfizer, Lilly. N.D. reports receiving honoraria from BMS, Merck and receiving support for congress participation from BMS. M.C.M. reports receiving honoraria from AstraZeneca, BMS, Merck Sharp \& Dohme, Merck and acting as consultant for Merck.

T.G., E.B., L.D’O., A.B., M.P., P.V., C.S., L.C., M.E.C., F.R., E.C., G.A., D.S., L.B., R.A., E.D.C., M.A. report no financial competing interest.

\section{Ethical approval}

The study was approved by the Comitato Etico Interaziendale ASO S. Croce e Carle Cuneo, AA SS LL CN1, CN2, AT. The study was performed in accordance with the ethical standards as laid down in the 1964 Declaration of Helsinki and its later amendments or comparable ethical standards

\section{Informed Consent}

Informed consent was obtained for all participants

\section{References}

1. Ross JS, Slodkowska EA, Symmans WF, Pusztai L, Ravdin PM, Hortobagyi GN (2009) The HER-2 receptor and breast cancer: ten years of targeted anti-HER-2 therapy and personalized medicine. Oncologist 14(4):320-368

2. Wolff AC, Hammond MEH, Hicks DG, Dowsett M, Mc Shane LM, Allred DC et al. Recommendations for human epidermal growth factor receptor 2 testing in breast cancer:

3. American Society of Clinical Oncology/ (2013) College of American Pathologists clinical practice guideline update. J Clin Oncol 31(31):3997-4013

4. Piccart-Gebhart MJ, Procter M, Leyland-Jones B, Goldhirsch A, Untch M, Smith I et al (2005) Trastuzumab after adjuvant chemotherapy in HER2-positive breast cancer. N Engl J Med 353(16):1659-1672 
5. Romond EH, Perez EA, Bryant J, Suman VJ, Geyer CE Jr, Davidson NE et al (2005) Trastuzumab plus adjuvant chemotherapy for operable HER2-posirtive breast cancer: combined analysis of NSABP B31 and NCCTG N9831. N Engl J Med 353:1673-1684

6. Slamon D, Eiermann W, Robert N, Pienkowski T, Martin M, Press M et al, et al (2011) Adjuvant trastuzumab in HER2-positive breast cancer. N Engl J Med 365(14):1273-1283

7. Slamon DJ, Leyland-Jones B, Shak S, Fuchs H, Paton V, Bajamonde A et al (2001) Use of chemotherapy plus a monoclonal antibody against HER2 for metastatic breast cancer that overexpresses HER2. N Engl J Med 344(11):783-792

8. Moja L, Tagliabue L, Balduzzi S, Parmelli E, Pistotti V, Guarneri V et al. Trastuzumab containing regimens for early breast cancer. Cochrane Database Syst Rev. 2012 Apr 18;2012(4):CD006243. doi: 10.1002/14651858.CD006243.pub2

9. Balduzzi S, Mantarro S, Guarneri V, Tagliabue L, Pistotti V, Moja L et al. Trastuzumab containing regimens for metastatica breast cancer. Cochrane Database Syst Rev. 2014 Jun 12;2014(6):CD006242. doi: 10.1002/14651858.CD006242.pub2

10. Baselga J, Cortés J, Kim SB, Im SA, Hegg R, Im YH et al (2012) Pertuzumab plus trastuzumab plus docetaxel for metastatic breast cancer. N Engl J Med 366(2):109-119

11. Swain SM, Baselga J, Kim SB, Ro J, Semiglazov V, Campone M et al (2015) Pertuzumab, trastuzumab, and docetaxel in HER2-positive metastatic breast cancer. N Engl J Med 372:724-734

12. Swain SM, Miles D, Kim SB, Im YH, Im SA, Semiglazov V et al (2020) End-of-study analysis from the phase III, randomized, double-blind, placebo (Pla)-controlled CLEOPATRA study of first-line (1L) pertuzumab $(P)$, trastuzumab $(H)$, and docetaxel $(D)$ in patients $(p t s)$ with HER-2positive metastatic breast cancer (MBC). Lancet Oncol 21(4):519-530

13. Dang C, lyengar N, Datko F, D'Andrea G, Theodoulou M, Dickler M et al (2015) phase Il study of paclitaxel given once per week along with trastuzumab and pertuzumab in patients with human epidermal growth factor receptor 2-positive metastatic breast cancer. J Clin Oncol 33:442-447

14. Wang R, Smyth LM, lyengar N, Chandarlapaty S, Modi S, Jochelson M et al (2019) Phase II study of weekly paclitaxel with trastuzumab and pertuzumab in patients with human epidermal growth receptor 2 overexpressing metastatic breast cancer: 5-year follow-up. Oncologist 24:1-7

15. Bachelot T, Ciruelos E, Schneeweiss A, Puglisi F, Peretz-Yablonski T, Bondarenko I et al (2019 May) Preliminary safety and efficacy of first-line pertuzumab combined with trastuzumab and taxane therapy for HER2-positive locally recurrent or metastatic breast cancer (PERUSE). Ann Oncol 1;30(5):766-773

16. Eisenhauer EA, Therasse P, Bogaerts J, Schwartz LH, Sargent D, Ford R et al (2009) New response evaluation criteria in solid tumours; revised RECIST guideline (version 1.1). Eur J Cancer 45:228-247

17. Kaplan EL, Meier P (1958) Nonparametric estimation from incomplete observations. J Am Stat Assn 53:457-481

18. Peto R, Peto J (1972) Asymptotically efficient rank invariant test procedures. J Royal Stat Soc A 135(2):185-207 
19. Giuliano M, Hu H, Wang Y-C, Fu X, Nardone A, Herrera S et al (2015) Upregulation of ER Signaling as an adaptive mechanism of cell survival in HER2-Positive breast tumors treated with anti-HER2 therapy. Clin Cancer Res 21(17):3995-4003

20. Puglisi F, Minisini AM, De Angelis C, Arpino G (2012) Overcoming treatment resistance in HER2positive breast cancer: Potential strategies. Drugs 72:1175-1193

21. Rimawi M, Ferrero JM, de la Haba-Rodriguez J, Poole C, De Placido S, Osborne CK et al (2018) First line trastuzumab plus an aromatase inhibitor, with or without pertuzumab, in human epidermal growth factor receptor-2 positive and hormone receptor metastatic or locally advanced breast cancer (PERTAIN): a randomized, open-label phase II trial. J Clin Oncol 36(8):741-748

22. De Placido S, Giuliano M, Schettini F, Von Arx C, Buono G, Riccardi F et al (2018) Human epidermal growth factor receptor 2 dual blockade with trastuzumab and pertuzumab in real life: Italian clinical practice versus the CLEOPATRA trial results. The Breast 38:86-91

23. Gamucci T, Pizzuti L, Natoli C, Mentuccia L, Sperduti I, Barba M et al (2019) A multicenter retrospective observational study of first-line treatment with pertuzumab, trastuzumab and taxanes for advanced HER2 positive breast cancer patients. RePer Study Cancer Biology Therapy 20(2):192200

24. Cardoso F, Paluch-Schimon S, Senkus E, Curigliano G, Aapro M, André F et al (2020) 5th ESO-ESMO international consensus guideline for advanced breast cancer (ABC 5). Ann Oncol. doi:https://doi.org/10.1016/j.annonc.2020.09.010

25. Robert NJ, Goeertz H-P, Chopra P, JiaoX, Yoo B, Patt D et al (2017) Her2 positive metastatic breast cancer patients receiving pertuzumab in a community oncology practice setting: treatment patterns and outcomes. Drugs Real World Outcomes 4:1-7

26. Perez AE, Lopez-Vega JM, Petit T, Zamagni C, Easton V, Kamber J et al (2016) Safety and efficacy of vinorelbine in combination with pertuzumab and trastuzumab for first-line treatment of patients with HER2-positive locally advanced or metastatic breast cancer: VELVET Cohort 1 final results. Breast Cancer Res 18:126. doi:10.1186/s13058-016-0773-6

27. Miles D, Im YH, Fung A, Yoo B, Knott A, Heeson S et al (2017) Effect of docetaxel duration on clinical outcomes: exploratory analysis of CLEOPATRA, a phase III randomized controlled trial. Ann Oncol 28(11):2761-2767

28. Garrone O, Montemurro F, Saggia C, La Verde N, Vandone AM, Airoldi M et al (2016) Eribulin in pretreated metastatic breast patients: results of the TROTTER trial- a multicenter retrospective study of eribulin in real life. Springerplus 5:59. DOI 10.1186/s40064-016-1700-0

29. Vici P, Pizzuti L, Michelotti A, Sperduti I, Natoli C, Mentuccia L et al (2017) A retrospective multicentric observational study of trastuzumab emtansine in HER2 positive metastatic breast cancer: a realworld experience. Oncotarget 8(34):56921-56931

30. Cazzaniga ME, Airoldi M, Arcangeli V, Artale S, Atzori F, Ballerio A et al (2017) Efficacy and safety of everolimus and exemestane in hormone-receptor positive (HR+) human-epidermal-growth-factor 
negative (HER2-) advanced breast cancer patients: new insights beyond clinical trials. The EVA study. Breast 35:115-121

Figures

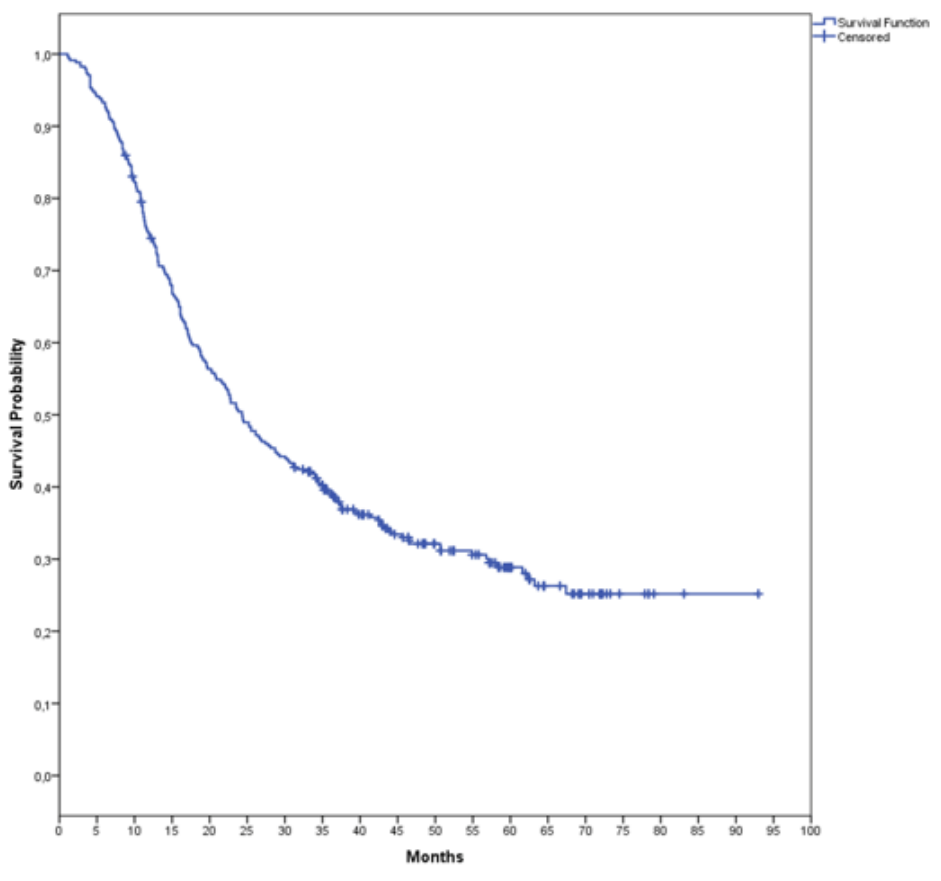

a

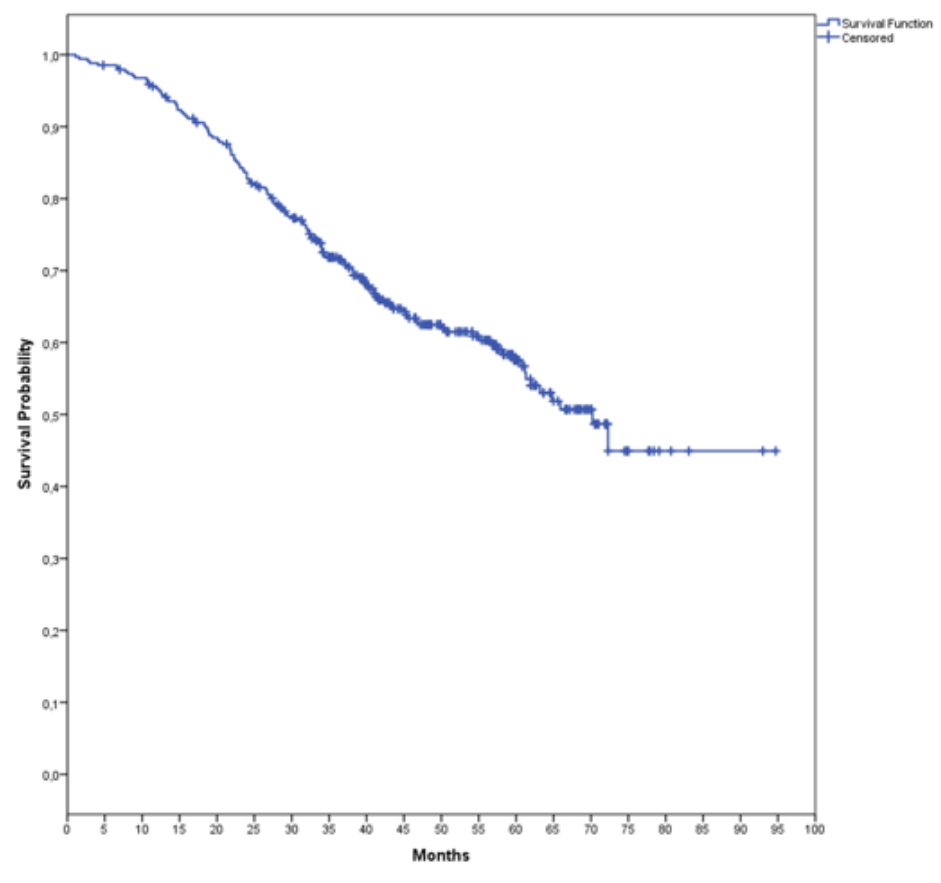

b

\section{Figure 1}

Progression-Free Survival (a) and Overall Survival (b) in the overall population

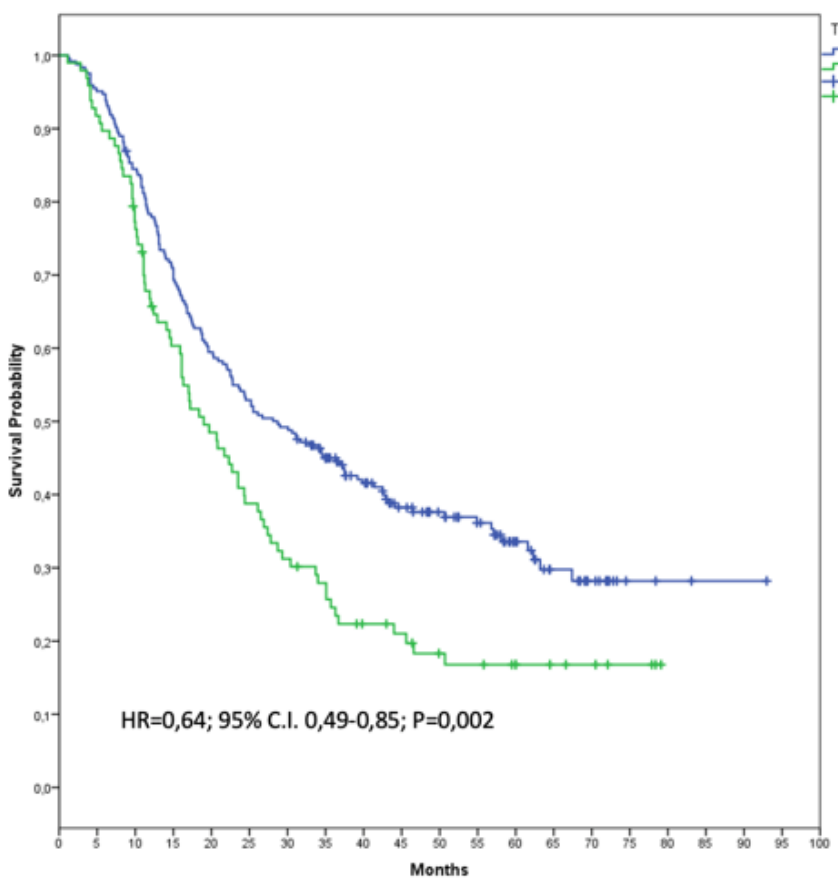

a

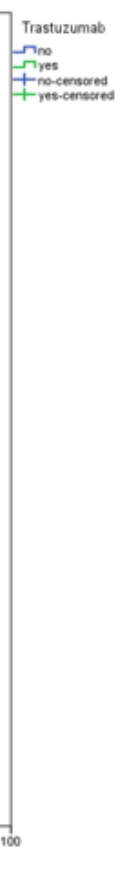

Page $16 / 17$

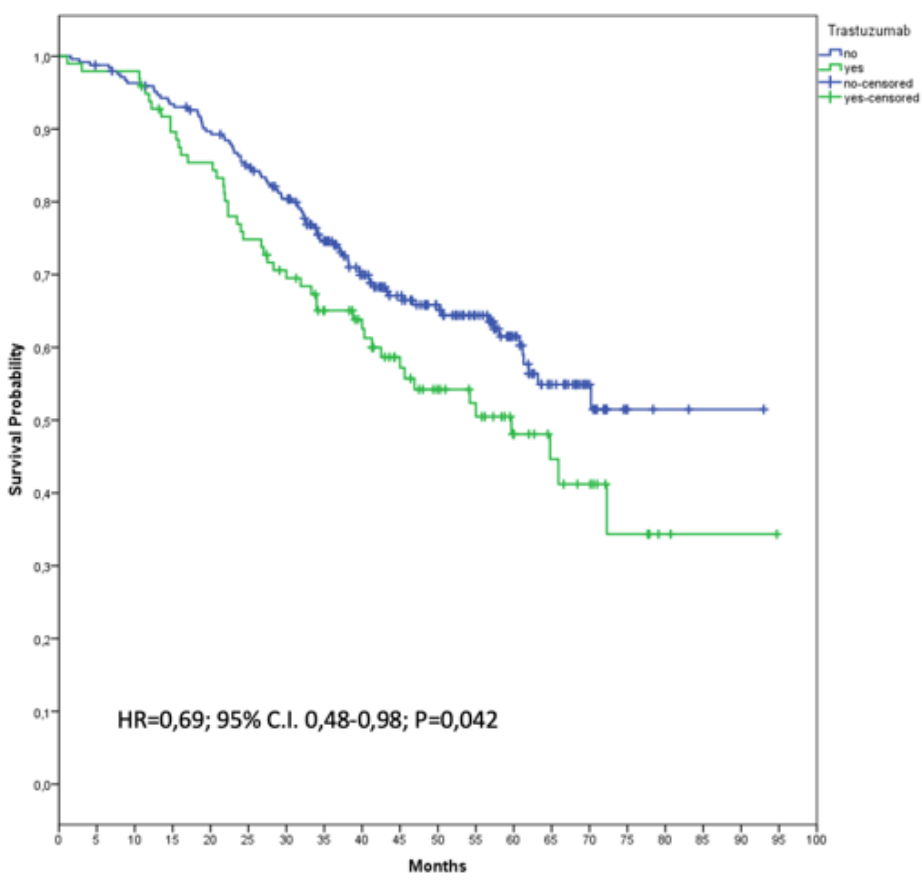

b 
Figure 2

Progression-Free Survival (a) and Overall Survival (b) according to exposure to adjuvant trastuzumab 